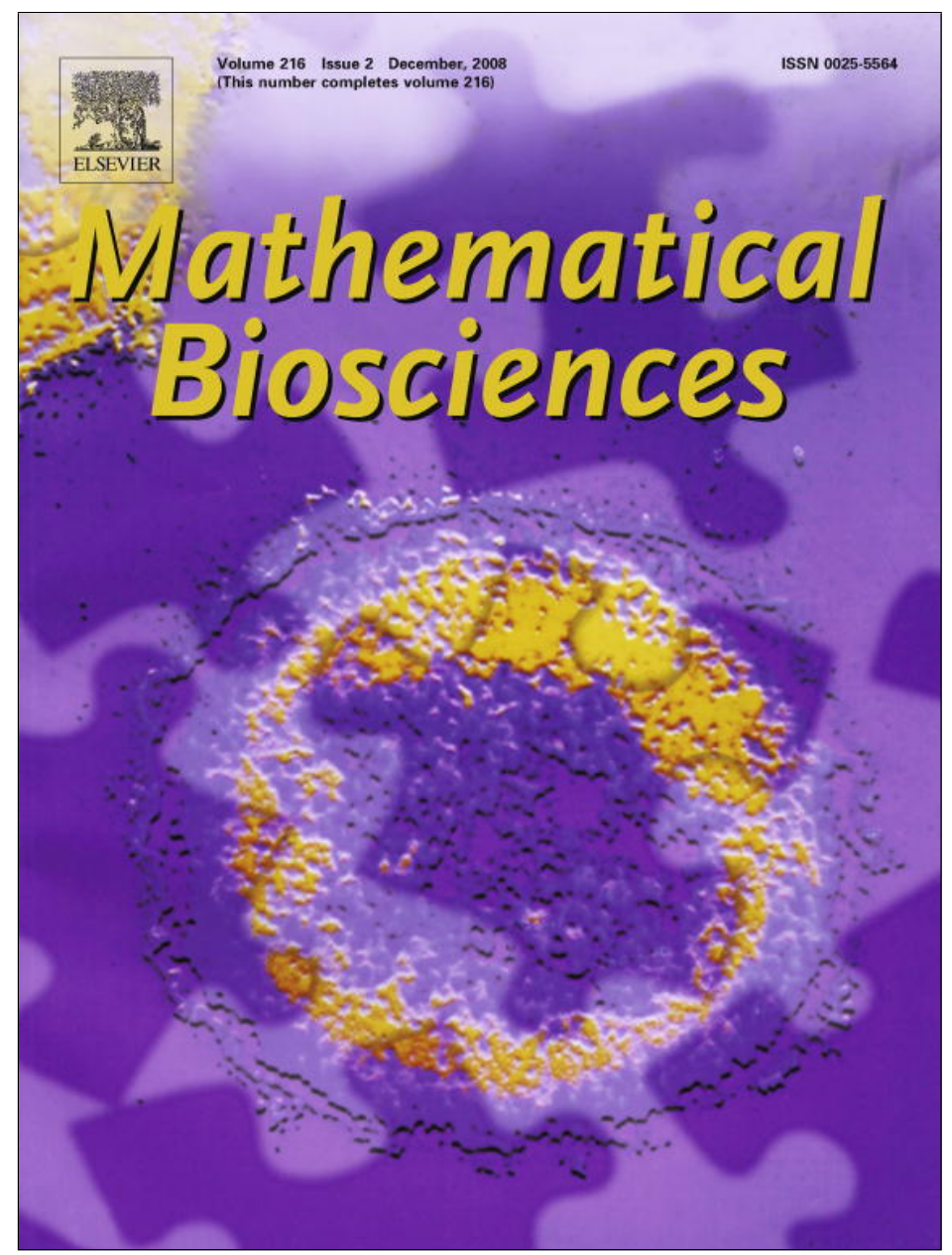

This article appeared in a journal published by Elsevier. The attached copy is furnished to the author for internal non-commercial research and education use, including for instruction at the authors institution and sharing with colleagues.

Other uses, including reproduction and distribution, or selling or licensing copies, or posting to personal, institutional or third party websites are prohibited.

In most cases authors are permitted to post their version of the article (e.g. in Word or Tex form) to their personal website or institutional repository. Authors requiring further information regarding Elsevier's archiving and manuscript policies are encouraged to visit:

http://www.elsevier.com/copyright 


\title{
Yakubovich's oscillatority of circadian oscillations models
}

\author{
Denis V. Efimov*, Alexander L. Fradkov \\ Control of Complex Systems Laboratory, Institute of Problem of Mechanical Engineering, Bolshoi Avenue, 61, V.O., St-Petersburg 199178, Russia
}

\section{A R T I C L E I N F O}

\section{Article history:}

Received 22 August 2007

Received in revised form 27 June 2008

Accepted 2 October 2008

Available online 14 October 2008

\section{Keywords:}

Biological systems

Lyapunov methods

Nonlinear oscillators

Oscillator stability

\begin{abstract}
A B S T R A C T
The testing procedure of Yakubovich's oscillatority property is presented. The procedure is applied for two models of circadian oscillations [J.C. Leloup, A. Goldbeter, A model for circadian rhythms in Drosophila incorporating the formation of a complex between the PER and TIM proteins, J. Biol. Rhythms, 13 (1998) 70-87; J.C. Leloup, D. Gonze, A. Goldbeter, Limit cycle models for circadian rhythms based on transcriptional regulation in Drosophila and Neurospora. J. Biol. Rhythms, 14 (1999) 433-448]. Analytical conditions of these models oscillatority are established and bounds on oscillation amplitude are calculated.
\end{abstract}

(c) 2008 Elsevier Inc. All rights reserved.

\section{Introduction}

During recent years an interest in studying more complex behavior of the systems related to oscillatory and chaotic modes has grown significantly. It was founded that important and useful concept for studying irregular oscillations in dynamical systems is "oscillatority" introduced by V.A.Yakubovich in 1973 [14]. Frequency domain conditions for oscillatority were obtained for Lurie systems, composed on linear and nonlinear parts $[12,14,15]$. Oscillation analysis and design methods for generic nonlinear systems were proposed in [5]. The result of [5] was developed in [6] for nonlinear time delay systems. In [6] the proposed results were applied to several biological systems, which models can be described by nonlinear dynamical equations with delays. Among them the model of circadian rhythms in Drosophila from works $[8,9]$ was analyzed. The considered model from [8] has dimension of five. In work [10] more detailed model of circadian oscillations was proposed (with dimension 10), that incorporates the formation of a complex between the PER and TIM proteins. In paper [11] it was noted that circadian oscillations in Drosophila and Neurospora are closely related by the nature of the feedback loop that governs circadian rhythmicity, even if they differ by the identity of the molecules involved in the regulatory circuit. The simple model of circadian oscillation in Neurospora was presented in [11] (with dimension 3).

\footnotetext{
is This work is partly supported by Grant 05-01-00869 of Russian Foundation for Basic Research, by Russian Science Support Foundation and by Program of Presidium of Russian Academy of Science No. 19.

* Corresponding author. Present address: Montefiore Inst., B28 Université de Liège, 4000, Belgium.
}

E-mail addresses: efde@mail.ru (D.V. Efimov), alf@control.ipme.ru (A.L. Fradkov).
In this paper the theory developed in [5,6] is applied to the models of circadian oscillations in Drosophila and Neurospora from papers [10] and [11] to derive conditions of oscillations arising in the systems. This topic of research dealing with conditions of oscillatority of various circadian rhythms models is very popular in the last years [16-19] (just to mention the latest papers). Mainly the researches in this field are oriented on developing conditions of periodical oscillations existence that results to rather complex and local analysis of the models. The concept of Yakubovich's oscillatority covers any types of irregular oscillations as well as periodical ones (without distinguishing the type of periodicity of oscillating modes). Such relaxation allows one to simplify the testing conditions, additionally, the conditions provide the restrictions on all admissible values of parameters ensuring oscillatority for the model (in contrast with bifurcation approach [16], where existence of oscillations are guaranteed only locally in the vicinity of bifurcation point, while values of parameters of real biological processes can be far beyond the bifurcation).

In the following section some definitions and notations from [5] are introduced and the procedure for oscillatority property establishing is formulated. In section 3 the model of circadian oscillations in Drosophila from [11] is considered. In section 4 the complex model of circadian rhythms in Drosophila from [10] is analyzed.

\section{Preliminaries}

Let us consider the following model of nonlinear dynamical system:

$$
\dot{\mathbf{x}}=\mathbf{f}(\mathbf{x})
$$


where $\mathbf{x} \in R^{n}$ is the state space vector; $\mathbf{f}$ is locally Lipschitz continuous function on $R^{n}, \mathbf{f}(0)=0$. Solution $\mathbf{x}\left(\mathbf{x}_{0}, t\right)$ of the system (1) with initial condition $\mathbf{x}_{0} \in R^{n}$ is defined at the least locally for $t \leqslant T$ (further we will simply write $\mathbf{x}(t)$ if initial conditions are clear from the context). If $T=+\infty$ for all initial conditions, then such system is called forward complete.

As usual, function $\rho: R_{+} \rightarrow R_{+}$belongs to class $K$, if it is strictly increasing and $\rho(0)=0 ; \rho \in K_{\infty}$ if $\rho \in K$ and $\rho(s) \rightarrow \infty$ for $s \rightarrow \infty ; R_{+}=\{\tau \in R: \tau \geqslant 0\}$. Notation $D V(\mathbf{x}) \mathbf{F}(\cdot)$ stands for directional derivative of function $V$ with respect to vector field $\mathbf{F}$ if function $V$ is differentiable and for Dini derivative in the direction of $\mathbf{F}$

$D V(\mathbf{x}) \mathbf{F}(\cdot)=\lim _{t \rightarrow 0^{+}} \inf \frac{V(\mathbf{x}+t \mathbf{F}(\cdot))-V(\mathbf{x})}{t}$

if function $V$ is Lipschitz continuous.

Definition 1 [5]. Solution $\mathbf{x}\left(\mathbf{x}_{0}, t\right)$ with $\mathbf{x}_{0} \in R^{n}$ of system (1) is called $\left[\pi^{-}, \pi^{+}\right]$-oscillation with respect to output $\psi=\eta(\mathbf{x})$ (where $\eta: R^{n} \rightarrow R$ is a continuous monotonous with respect to all arguments function) if the solution is defined for all $t \geqslant 0$ and

$\varliminf_{t \rightarrow+\infty} \psi(t)=\pi^{-} ; \varlimsup_{t \rightarrow+\infty} \psi(t)=\pi^{+} ;-\infty<\pi^{-}<\pi^{+}<+\infty$.

Solution $\mathbf{x}\left(\mathbf{x}_{0}, t\right)$ with $\mathbf{x}_{0} \in R^{n}$ of system (1) is called oscillating, if there exist some output $\psi$ and constants $\pi^{-}, \pi^{+}$such, that $\mathbf{x}\left(\mathbf{x}_{0}, t\right)$ is $\left[\pi^{-}, \pi^{+}\right]$-oscillation with respect to the output $\psi$. Forward complete system (1) is called oscillatory, if for almost all $\mathbf{x}_{0} \in R^{n}$ solutions of the system $\mathbf{x}\left(\mathbf{x}_{0}, t\right)$ are oscillating. Oscillatory system (1) is called uniformly oscillatory, if for almost all $\mathbf{x}_{0} \in R^{n}$ for corresponding solutions $\mathbf{x}\left(\mathbf{x}_{0}, t\right)$ there exist output $\psi$ and constants $\pi^{-}, \pi^{+}$non-depending on initial conditions.

Note that term "almost all solutions" is used to emphasize that generally system (1) has a nonempty set of equilibrium points, thus, there exists a set of initial conditions with zero measure such, that corresponding solutions are not oscillations. It is worth to stress, that constants $\pi^{-}$and $\pi^{+}$are exact asymptotic bounds for output $\psi$. Conditions of oscillation existence in the system are summarized in the following theorem.

Theorem 1 [5]. Let system (1) have two continuous and locally Lipschitz Lyapunov functions $V_{1}$ and $V_{2}$ satisfying for all $\mathbf{x} \in R^{n}$ and $t \in R_{+}$inequalities:

$v_{1}(|\mathbf{x}|) \leqslant V_{1}(\mathbf{x}, t) \leqslant v_{2}(|\mathbf{x}|), v_{3}(|\mathbf{x}|) \leqslant V_{2}(\mathbf{x}, t) \leqslant v_{4}(|\mathbf{x}|)$,

for $v_{1}, v_{2}, v_{3}, v_{4} \in K_{\infty}$ and

$\partial V_{1} / \partial t+D V_{1}(\mathbf{x}, t) \mathbf{f}(\mathbf{x})>0$ for $0<|\mathbf{x}|<X_{1}$ and $\mathbf{x} \notin \Xi ;$

$\partial V_{2} / \partial t+D V_{2}(\mathbf{x}, t) \mathbf{f}(\mathbf{x})<0$ for $|\mathbf{x}|>X_{2}$ and $\mathbf{x} \notin \Xi$,

$X_{1}<v_{1}^{-1} \circ v_{2} \circ v_{3}^{-1} \circ v_{4}\left(X_{2}\right)$,

where $\Xi \subset R^{n}$ is a set with zero Lebesgue measure, and $\Omega \cap \Xi$ is empty set, $\Omega=\left\{\mathbf{x}: v_{2}^{-1} \circ v_{1}\left(X_{1}\right)<|\mathbf{x}|<v_{3}^{-1} \circ v_{4}\left(X_{2}\right)\right\}$. Then the system is oscillatory.

Note, that the set $\Omega$ determines lower bound for value of $\pi^{-}$and upper bound for value of $\pi^{+}$.

Like in [15] one can consider Lyapunov function for linearized near the origin system (1) as a function $V_{1}$ to prove local instability of the system solutions. Instead of existence of Lyapunov function $V_{2}$ one can require just boundedness of the system solution $\mathbf{x}(t)$ with known upper bound. It can be obtained using another approach not dealing with time derivative of Lyapunov function analysis. In this case Theorem 1 transforms into Theorem 3.4 from [7].
Conditions of above theorem are rather general and define the class of systems, which oscillatory behavior can be investigated by the approach. Namely systems, which have in state space attracting compact set containing oscillatory movements of the systems. For such systems Theorem 1 gives the useful tool for testing oscillating behavior and obtaining estimates for the amplitude of oscillations. It is possible to show that for a subclass of uniformly oscillating systems proposed conditions are also necessary.

Theorem 2 [4]. Let system (1) be uniformly oscillatory with respect to the output $\psi=\eta(\mathbf{x})$ (where $\eta: R^{n} \rightarrow R$ is a continuous monotonous with respect to all arguments function), and for all $\mathbf{x} \in R^{n}$ the following relations are satisfied:

$\chi_{1}(|\mathbf{x}|) \leqslant \eta(\mathbf{x}) \leqslant \chi_{2}(|\mathbf{x}|), \chi_{1}, \chi_{2} \in K_{\infty} ;$

the set of initial conditions for which system is not oscillating consists in just one point $\Xi=\{\mathbf{x}: \mathbf{x}=0\}$. Then there exist two continuous and locally Lipschitz Lyapunov functions $V_{1}: R^{n+1} \rightarrow R_{+}$and $V_{2}: R^{n+1} \rightarrow R_{+}$such, that for all $\mathbf{x} \in R^{n}$ and $t \in R_{+}$inequalities hold:

$v_{1}(|\mathbf{x}|) \leqslant V_{1}(\mathbf{x}, t) \leqslant v_{2}(|\mathbf{x}|)$,

$v_{3}(|\mathbf{x}|) \leqslant V_{2}(\mathbf{x}, t) \leqslant v_{4}(|\mathbf{x}|), v_{1}, v_{2}, v_{3}, v_{4} \in K_{\infty} ;$

$\partial V_{1} / \partial t+D V_{1}(\mathbf{x}, t) \mathbf{f}(\mathbf{x})>0$ for $0<|\mathbf{x}|<\chi_{2}^{-1}\left(\pi^{-}\right) ;$

$\partial V_{2} / \partial t+D V_{2}(\mathbf{x}, t) \mathbf{f}(\mathbf{x})<0$ for $|\mathbf{x}|>\chi_{1}^{-1}\left(\pi^{+}\right)$.

For the uniformly oscillatory systems with single equilibrium point at the origin Theorems 1 and 2 give necessary and sufficient conditions of oscillations existence. According to the results of works [8-11] the circadian rhythms in Drosophila and Neurospora are the nice examples of uniformly oscillating systems. In this case application of proposed in Theorems 1 and 2 theory to the circadian oscillation models is natural for deriving conditions of oscillations existence. That is more expressions for time derivatives of Lyapunov functions $V_{1}$ and $V_{2}$ can provide analytical parametric conditions for oscillations existence.

Finally, let us describe the testing procedure of oscillatority property presence in dynamical nonlinear systems:

(1) calculation of equilibrium points coordinates;

(2) determining boundedness of the system trajectories property (using function $V_{2}$ or applying another approach);

(3) confirmation of local instability property in an equilibrium (applying function $V_{1}$ or using the first approximation of the system dynamics in the equilibrium);

(4) the form of set $\Omega$ calculation and verification of the equilibrium points absence in that set.

If all four steps are successfully passed, then the system is oscillatory in the sense of Yakubovich (Definition 1). Let us apply the above procedure to circadian oscillations models in Drosophila and Neurospora.

\section{Circadian oscillations in Neurospora}

Following [11] let us consider the following model of the oscillations:

$$
\begin{aligned}
& \dot{M}=v_{s} \frac{K_{I}^{n}}{K_{I}^{n}+F_{N}^{n}}-v_{m} \frac{M}{K_{m}+M} ; \\
& \dot{F}_{c}=k_{s} M-v_{d} \frac{F_{c}}{K_{d}+F_{c}}-k_{1} F_{c}+k_{2} F_{N} \\
& \dot{F}_{N}=k_{1} F_{c}-k_{2} F_{N}
\end{aligned}
$$


where variables $M, F_{c}$ and $F_{N}$ denote, respectively, the concentrations (defined with respect to the total cell volume) of the frq mRNA and of the cytosolic and nuclear forms of FRQ.

According to proposed procedure let us start with coordinates of equilibrium calculation for system (2)-(4). Equating to the zero the right-hand side of the system we obtain the following system of nonlinear equations:

$$
\begin{aligned}
F_{c}^{0} & =k_{2} k_{1}^{-1} F_{N}^{0} ; \quad M^{0}=\frac{v_{d} k_{2} F_{N}^{0}}{k_{s}\left(K_{d} k_{1}+k_{2} F_{N}^{0}\right)} ; \quad v_{s} \frac{K_{I}^{n}}{K_{I}^{n}+\left(F_{N}^{0}\right)^{n}} \\
& =v_{m} \frac{M^{0}}{K_{m}+M^{0}},
\end{aligned}
$$

where $M^{0}, F_{c}^{0}, F_{N}^{0}$ are coordinates of possible equilibriums. It is clear that for any positive values of the system (2)-(4) parameters the first two equations in Eq. (5) have the single positive solutions. The third equation also admits only single positive solution since the function on the left-hand side is strictly decreasing to zero, while the function on the right-hand side is strictly increasing from zero. Thus, the system has the single equilibrium with positive coordinates (strictly bigger than zero) for positive values of the parameters. For $n \leqslant 3$ it is possible to analytically calculate the values of $M^{0}, F_{c}^{0}, F_{N}^{0}$ as functions of the system parameters, for $n>3$ only numerical solution is possible in generic case.

As the second step let us base the global boundedness of the system trajectories property using the following propositions.

Proposition 1. Let $v_{s} \geqslant v_{m}$. Then there exists $\delta \in(0,1)$ such, that if for some $t_{0}>0$ it holds that

$F_{N}\left(t_{0}\right) \geqslant \varepsilon, F_{c}\left(t_{0}\right) \geqslant k_{2} k_{1}^{-1} \varepsilon$,

$M\left(t_{0}\right) \geqslant 2 \frac{v_{d}}{k_{s}}, \varepsilon=K_{I} \sqrt[n]{\frac{v_{s}}{v_{m}}\left(1+K_{m} \frac{k_{s}}{\delta v_{d}}\right)-1}$,

then there exists time instant $t_{1} \geqslant t_{0}$ such, that

$F_{N}(t) \leqslant \varepsilon, F_{c}(t) \leqslant k_{2} k_{1}^{-1} \varepsilon, M(t) \leqslant 2 \frac{v_{d}}{k_{s}}$ for all $t \geqslant t_{1}$.

Note that the case $v_{s}<v_{m}$ can be considered in the same way as it was analyzed for the five order model in paper [1]

As the third step let us investigate the conditions of the equilibrium instability using the first approximation of the system near the equilibrium:

$\mathbf{x}=\mathbf{A}\left(M^{0}, F_{c}^{0}, F_{N}^{0}\right) \mathbf{x}, \mathbf{A}\left(M^{0}, F_{c}^{0}, F_{N}^{0}\right)$

$$
=\left[\begin{array}{ccc}
-v_{m} \frac{K_{m}}{\left(K_{m}+M^{0}\right)^{2}} & 0 & \frac{-v_{s}\left(K_{I} F_{N}^{0}\right)^{n}}{\left(K_{I}^{n}+\left(F_{N}^{0}\right)^{n}\right)^{2}} \frac{n}{F_{N}^{0}} \\
k_{s} & \frac{-v_{d} K_{d}}{\left(K_{d}+F_{c}^{0}\right)^{2}}-k_{1} & k_{2} \\
0 & k_{1} & -k_{2}
\end{array}\right] \text {, }
$$

where $\mathbf{x}=\left(\delta M, \delta F_{c}, \delta F_{N}\right)^{T}$ is vector of the system state deviations from the equilibrium and $\mathbf{A}$ is the first approximation matrix dependent on the model parameters. The characteristic polynomial of the matrix $\mathbf{A}$ has form

$$
\begin{aligned}
& p(s)=s^{3}+a_{1} s^{2}+a_{2} s+a_{3}, \\
& \left(\begin{array}{l}
a_{3} \\
a_{2} \\
a_{1}
\end{array}\right)=\left(\begin{array}{c}
\frac{K_{m} k_{2} v_{m}}{\left(K_{m}+M_{0}\right)^{2}}\left(2 k_{1}+\frac{K_{d} v_{d}}{\left(F_{c}^{0}+K_{d}\right)^{2}}\right)-\frac{k_{1} k_{s} n v_{s}\left(F_{N}^{0} K_{I}\right)^{n}}{F_{N}^{0}\left(\left[F_{N}^{0}\right)^{n}+K_{l}^{n}\right]^{2}} \\
\left(k_{1}+\frac{K_{d} v_{d}}{\left(F_{c}^{0}+K_{d}\right)^{2}}\right)\left(k_{2}+\frac{K_{m} v_{m}}{\left(K_{m}+M_{0}\right)^{2}}\right)+k_{1} k_{2}+\frac{K_{m} k_{2} v_{m}}{\left(K_{m}+M_{0}\right)^{2}} \\
k_{1}+k_{2}+\frac{K_{d} v_{d}}{\left(F_{c}^{0}+K_{d}\right)^{2}}+\frac{K_{m} v_{m}}{\left(K_{m}+M_{0}\right)^{2}}
\end{array}\right) .
\end{aligned}
$$

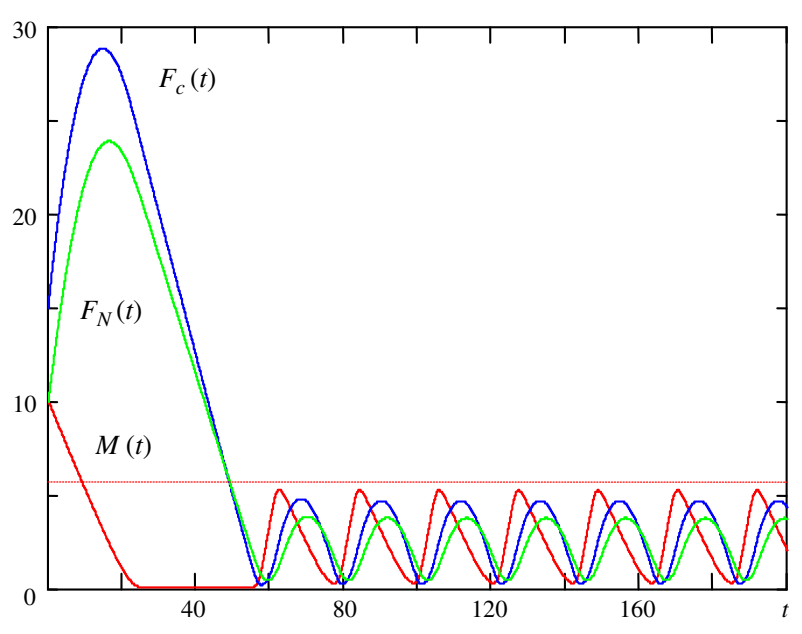

Fig. 1. Oscillations in model (2)-(4).

Applying Hurwitz criterion to the third order system (6) we obtain that, if the following inequalities are satisfied, then the matrix $\mathbf{A}$ has all eigen-values with negative real parts:

$a_{1}>0, \quad a_{1} a_{2}-a_{3}>0, \quad a_{3}>0$.

For any positive values of the parameters we have $a_{1}>0$, $a_{2}>0$, straightforward calculations show $a_{1} a_{2}-a_{3}>0$ (opening the brackets we obtain that all items have positive signs), thus the single possibility to lose the stability for Eq. (6) comes from coefficient $a_{3}$. The condition $a_{3}<0$ (which ensures for matrix $\mathbf{A}$ the presence of eigen-values with positive real parts) can be rewritten as follows:

$$
\begin{gathered}
k_{2} v_{m} K_{m}\left[v_{d} K_{d}+2 k_{1}\left(F_{c}^{0}+K_{d}\right)^{2}\right] F_{N}^{0}\left(K_{I}^{n}+\left(F_{N}^{0}\right)^{n}\right)^{2} \\
\ll k_{1} k_{s} n v_{s}\left(K_{I} F_{N}^{0}\right)^{n}\left(F_{c}^{0}+K_{d}\right)^{2}\left(K_{m}+M_{0}\right)^{2} .
\end{gathered}
$$

Inequality (7) is a function of the system parameters and the equilibrium coordinates $F_{N}^{0}, F_{c}^{0}, M^{0}$, which are the solution of equations (5). For $n \leqslant 3$ equations (5) have analytical solutions, substituting which in Eq. (7) it is possible to obtain system instability condition dependent on the parameters only.

The fourth step is obvious since the system (2)-(4) has only one equilibrium. Thus, the key condition of the system oscillatority is inequality (7). In [11] the model (2)-(4) was considered with the following values of parameters:

$$
\begin{aligned}
& v_{m}=0.505, \quad v_{d}=1.4, \quad v_{s}=1.6, \quad k_{s}=0.5 \\
& k_{1}=0.5, \quad k_{2}=0.6, \quad K_{I}=1, \quad K_{m}=0.5, \quad K_{d}=0.13, \quad n=4 .
\end{aligned}
$$

For these values system of equations (5) has the solution:

$M_{0}=2.583, F_{c}^{0}=1.55, F_{N}^{0}=1.291$,

for which inequality (7) is true. Therefore the system with these values of parameters is oscillatory. The results of the system simulation is shown in Fig. 1. The dash line corresponds to obtained in Proposition 1 bounds for oscillations amplitude $2 k_{s}^{-1} v_{d}$.

\section{Circadian oscillations in Drosophila}

Let us consider the model from paper [10]: 


$$
\begin{aligned}
& \dot{M}_{P}=v_{s p} \frac{K_{I P}^{n}}{K_{I P}^{n}+C_{N}^{n}}-v_{m P} \frac{M_{P}}{K_{m P}+M_{P}}-k_{d} M_{P} ; \\
& \dot{P}_{0}=k_{s P} M_{P}-V_{1 P} \frac{P_{0}}{K_{1 P}+P_{0}}+V_{2 P} \frac{P_{1}}{K_{2 P}+P_{1}}-k_{d} P_{0} ; \\
& \dot{P}_{1}=V_{1 P} \frac{P_{0}}{K_{1 P}+P_{0}}-V_{2 P} \frac{P_{1}}{K_{2 P}+P_{1}}--V_{3 P} \frac{P_{1}}{K_{3 P}+P_{1}}+V_{4 P} \frac{P_{2}}{K_{4 P}+P_{2}}-k_{d} P_{1} ; \\
& \dot{P}_{2}=V_{3 P} \frac{P_{1}}{K_{3 P}+P_{1}}-V_{4 P} \frac{P_{2}}{K_{4 P}+P_{2}}--k_{3} P_{2} T_{2}+k_{4} C-v_{d P} \frac{P_{2}}{K_{d P}+P_{2}}-k_{d} P_{2} ; \\
& \dot{M}_{T}=v_{s p} \frac{K_{I T}^{n}}{K_{I T}^{n}+C_{N}^{n}}-v_{m T} \frac{M_{T}}{K_{m T}+M_{T}}-k_{d} M_{T} ; \\
& \dot{T}_{0}=k_{S T} M_{T}-V_{1 T} \frac{T_{0}}{K_{1 T}+T_{0}}+V_{2 T} \frac{T_{1}}{K_{2 T}+T_{1}}-k_{d} T_{0} ; \\
& \dot{T}_{1}=V_{1 T} \frac{T_{0}}{K_{1 T}+T_{0}}-V_{2 T} \frac{T_{1}}{K_{2 T}+T_{1}}--V_{3 T} \frac{T_{1}}{K_{3 T}+T_{1}}+V_{4 T} \frac{T_{2}}{K_{4 T}+T_{2}}-k_{d} T_{1} ; \\
& \dot{T}_{2}=V_{3 T} \frac{T_{1}}{K_{3 T}+T_{1}}-V_{4 T} \frac{T_{2}}{K_{4 T}+T_{2}}--k_{3} P_{2} T_{2}+k_{4} C-v_{d T} \frac{T_{2}}{K_{d T}+T_{2}}-k_{d} T_{2} ; \\
& \dot{C}_{=}=k_{3} P_{2} T_{2}-k_{4} C-k_{1} C+k_{2} C_{N}-k_{d C} C ; \\
& \dot{C}_{N}=k_{1} C-k_{2} C_{N}-k_{d N} C_{N},
\end{aligned}
$$

where $M_{P}$ is cytosolic concentration of per mRNA; $P_{0}, P_{1}, P_{2}$ are unphosphorylated, monophosphorylated and bisphosphorylated concentrations of PER protein correspondingly; $M_{T}$ is cytosolic concentration of tim mRNA; $T_{0}, T_{1}, T_{2}$ are unphosphorylated, monophosphorylated and bisphosphorylated concentrations of TIM protein correspondingly; $C$ is PER-TIM complex concentration and $C_{N}$ is nuclear form of PER-TIM complex. As in work [10] we will consider the following values of model (8)-(17) parameters:

$$
\begin{aligned}
& K_{I P}=K_{I T}=v_{s T}=v_{S P}=1, \quad v_{m P}=v_{m T}=0.7, \\
& K_{d P}=K_{d T}=K_{m P}=K_{m T}=0.2, \quad k_{S P}=k_{S T}=0.9, \\
& v_{d P}=v_{d T}=2, \quad V_{1 P}=V_{1 T}=V_{3 P}=V_{3 T}=8, \\
& K_{1 P}=K_{1 T}=K_{2 P}=K_{2 T}=K_{3 P}=K_{3 T}=K_{4 P}=K_{4 T}=2, \\
& k_{1}=0.6, \quad k_{2}=0.2, \quad k_{3}=1.2, \quad k_{4}=0.6, \quad n=4, \\
& k_{d}=k_{d C}=k_{d N}=0.01, \quad V_{2 P}=V_{2 T}=V_{4 P}=V_{4 T}=1 .
\end{aligned}
$$

Let us start with equilibriums number and their coordinates calculations. As in the model (2)-(4) for $n=4$ only numerical solutions of this problem is possible which shows that the system has the single equilibrium with coordinates:

$$
\begin{array}{llll}
M_{P}^{0}=1.513, & P_{0}^{0}=0.48, & P_{1}^{0}=0.469, & P_{2}^{0}=0.403, \\
M_{T}^{0}=1.513, & T_{0}^{0}=0.48, & T_{1}^{0}=0.469, & T_{2}^{0}=0.403, \\
C^{0}=0.305, & C_{N}^{0}=0.872 . &
\end{array}
$$

As the second step to prove boundedness of the system (8)-(17) trajectories it is possible to use the following Lyapunov function:

$$
\begin{aligned}
& V_{2}=P_{t}+T_{t}+2 \frac{k_{s P}}{k_{d}} M_{P}+2 \frac{k_{s T}}{k_{d}} M_{T}, \\
& P_{t}=P_{0}+P_{1}+P_{2}+C+C_{N}, T_{t}=T_{0}+T_{1}+T_{2}+C+C_{N},
\end{aligned}
$$

where $P_{t}$ and $T_{t}$ are total concentrations of PER and TIM proteins correspondingly. Time derivative of function $V_{2}$ admits the following upper estimate:

$\dot{V}_{2} \leqslant-0.5 \kappa V_{2}+2 \frac{k_{s P}}{k_{d}} v_{s P}+2 \frac{k_{s T}}{k_{d}} v_{s T}, \kappa=\min \left\{k_{d}, k_{d C}\right\}$,

which implies global boundedness of the system trajectories.

For the third step to establish local instability of the system equilibrium we use the first approximation of the system dynamics near the equilibrium. For the given values of the system

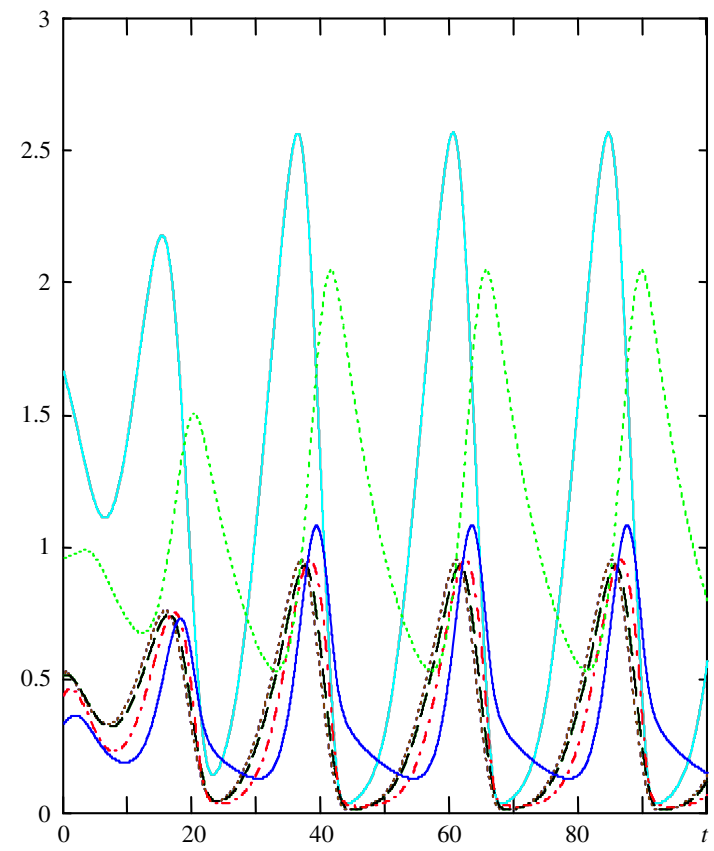

Fig. 2. Oscillations in model (8)-(17).

parameters the matrix of the first approximation has two complex conjugate eigen-values with positive real parts, that confirms local instability property of the equilibrium (we exclude the expressions of the matrix and its eigen-values for brevity of presentation).

Since the system has only one equilibrium the fourth step in this example is redundant and the system is oscillatory. The result of the system computer simulation is shown in Fig. 2.

\section{Conclusion}

The proposed in papers $[5,6]$ conditions of Yakubovich's oscillatority and presented here procedure of these conditions approving can be applied for wide range of biological systems (see examples in those papers and above). The approach also can be applied in adjacent areas like chemistry. The main advance of the solution comparing it with other approaches $[2,3,13]$ consists in simplicity of its application for generic class of nonlinear systems. As a side of results the bounds on oscillation amplitude can be calculated. Oscillatority in the sense of Yakubovich covers wide range of nonlinear oscillation behavior (from periodical to chaotic oscillations). The conditions are necessary and sufficient for some classes of oscillating systems like models of circadian oscillations considered in the paper.

\section{References}

[1] D Angeli, E.D Sontag, An analysis of circadian model using the small-gain approach to monotone systems, in: Proceedings of the 43rd IEEE Conference on Decision and Control, Nassau, Bahamas, 2004, pp. 575-580.

[2] V.I. Arnold, V.V. Kozlov, A.I. Neishtadt, Mathematical Aspects of Classical and Celestial Mechanics, Springer, Berlin, 1997.

[3] N.N. Bogolyubov, Y.A. Mitropolsky, Asymptotic Methods in the Theory of Nonlinear Oscillations, Gordon \& Breach, New York, 1962.

[4] D.V. Efimov Robust, Adaptive Control of Nonlinear Oscillations, SaintPetersburg, Nauka, 2005.

[5] D.V. Efimov, A.L. Fradkov, Excitation of oscillations in nonlinear systems under static feedback, in: Proceedings of the 43rd IEEE Conference on Decision and Control, Nassau, Bahamas, 2004, pp. 2521-2526.

[6] D.V. Efimov, A.L. Fradkov, Oscillatority conditions for nonlinear systems with delay, in: Proceedings of the 44th CDC-ECC 2005, Seville, 2005, pp. 6245-6250.

[7] A.L. Fradkov, A. Yu, Pogromsky Introduction to Oscillations and Chaos, World Scientific, Singapore, 1998. 
[8] A. Goldbeter, A model for circadian oscillations in the Drosophila period protein (PER), Proc. Roy. Soc. Lond. B 261 (1995) 319.

[9] A. Goldbeter, Biochemical Oscillations and Cellular Rhythms, Cambridge University, Cambridge, 1996.

[10] J.C. Leloup, A. Goldbeter, A model for circadian rhythms in Drosophila incorporating the formation of a complex between the PER and TIM proteins, J. Biol. Rhythms 13 (1998) 70.

[11] J.C. Leloup, D. Gonze, A. Goldbeter, Limit cycle models for circadian rhythms based on transcriptional regulation in Drosophila and Neurospora, J. Biol. Rhythms 14 (1999) 433.

[12] G.A. Leonov, I.M. Burkin, A.I. Shepelyavyi, Frequency Methods in Oscillation Theory, Kluwer, Dordrecht, 1995 (in Russian: 1992).

[13] J. Mallet-Paret, G.R. Sell, The Poincaré-Bendixson Theorem for monotone cyclic feedback systems with delay, J. Differential Equations 125 (1996) 441.
[14] V.A. Yakubovich, Frequency oscillations conditions in nonlinear systems with single stationary nonlinearity, Siberian Math. J. 14 (1973) 12.

[15] V.A. Yakubovich, Oscillations in systems with discontinuous and hysteresis nonlinearities, Automat. Remote Control 12 (1975).

[16] K. Tsumoto, T. Yoshinaga, H. Iida, H. Kawakami, K. Aihara, Bifurcations in a mathematical model for circadian oscillations of clock genes, J. Theor. Biol. 239 (1) (2006) 101.

[17] D. Savić, S. A Jelić, Mathematical model of the hypothalamo-pituitary adrenocortical system and its stability analysis, Chaos, Solitons \& Fractals 26 (2) (2005) 427.

[18] R. Wanga, L. Chena, K. Aihara, Detection of cellular rhythms and global stability within interlocked feedback systems, Math. Biosci. 209 (1) (2007) 171.

[19] Z. Xie, D. Kulasiri, Modelling of circadian rhythms in Drosophila incorporating the interlocked PER/TIM and VRI/PDP1 feedback loops, J. Theor. Biol. 245 (2) (2007) 290 